\title{
Melody Slot Machine
}

\author{
A Controllable Holographic Virtual Performer \\ Masatoshi Hamanaka \\ Center for Advanced Intelligence Project (AIP) \\ RIKEN \\ Tokyo, Japan \\ masatoshi.hamanaka@riken.jp
}

\begin{abstract}
This paper describes the "Melody Slot Machine," an interactive music system that enables control over virtual performers. Conventional virtual players focus on what kind of output performance is given to the input performance, and the performance output is difficult to control. The Melody Slot Machine enables the user to select the melody to be played next by the virtual player by rotating a dial. Furthermore, the performer is projected on a holographic display, and the user can feel as if a real virtual player is there. To achieve this, the system needs to change the melody and the performance video.
\end{abstract}

\section{CCS CONCEPTS}

- Applied computing - Arts and humanities • Sound and music computing

KEYWORDS: Melody morphing method; a generative theory of tonal music; holographic display

\section{ACM Reference format:}

Masatoshi Hamanaka. 2019. Melody Slot Machine: A Controllable Holographic Virtual Performer. In Proceedings of the 27th ACM Int'l Conference in Multimedia (MM'19), Oct. 21-25, 2019, Nice, France. ACM, New York, NY, USA. 8 pages. https://doi.org/10.1145/3343031.3350863

\section{INTRODUCTION}

For over 15 years, we have been studying musical operations based on the generative theory of tonal music (GTTM) [1-4]. Our goal is to develop a virtual musician that can compose and perform as well as a human being using the GTTM. Although there are still many challenges in achieving this, we used our melody-morphing method based on GTTM to develop a Melody Slot Machine and analyzed the tool at its current stage of

Permission to make digital or hard copies of all or part of this work for personal or classroom use is granted without fee provided that copies are not made or distributed for profit or commercial advantage and that copies bear this notice and the full citation on the first page. Copyrights for components of this work owned by others than ACM must be honored. Abstracting with credit is permitted. To copy otherwise, or republish, to post on servers or to redistribute to lists, requires prior specific permission and/or a fee. Request permissions from Permissions@acm.org.

MM '19, October 21-25, 2019, Nice, France

(C) 2019 Association for Computing Machinery.

ACM ISBN 978-1-4503-6889-6/19/10...\$15.00

https://doi.org/10.1145/3343031.3350863 development in terms of affecting a future virtual musician's appearance.

Our Melody Slot Machines provides a unique experience by enabling control of a virtual performer. The Melody Slot Machine has the following three features:

User-Friendly Interface We added a dial-type interface that can replace part of a melody segment that will be played by the virtual performer (Figure 1), allowing the user to easily control the virtual performer. The user's fingers can operate it on a tablet through a rectangular hole in part of the acrylic board, which is sandwiched between the score and the dial interface. When the red lever on the right side of the score is pulled down, all of the dials rotate, and one of the melody segments on the dial is randomly selected. Variations in melody segments are composed based on the generative theory of tonal music (GTTM) [1], so switching the melody segments maintains the overall structure of the melody and only changes the ornamentation.

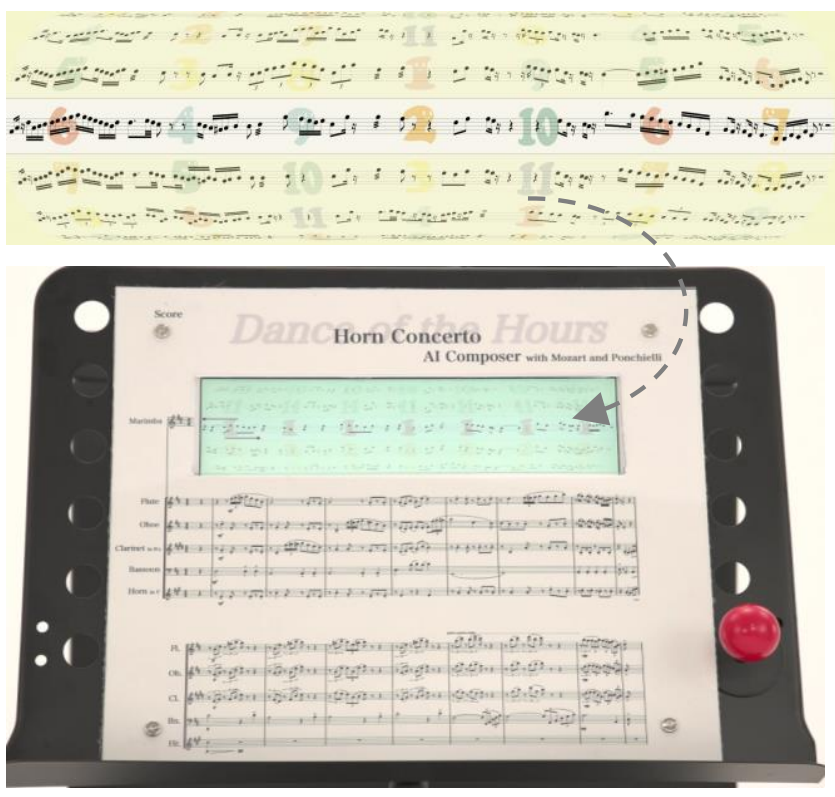

Figure 1: Slot dial and lever

Easy-to-Understand Control Results To confirm the results of the control visually as well as aurally, we prepared a 
holographic display that shows a virtual performer. This creates the sense of there being a presence (Figure 2).

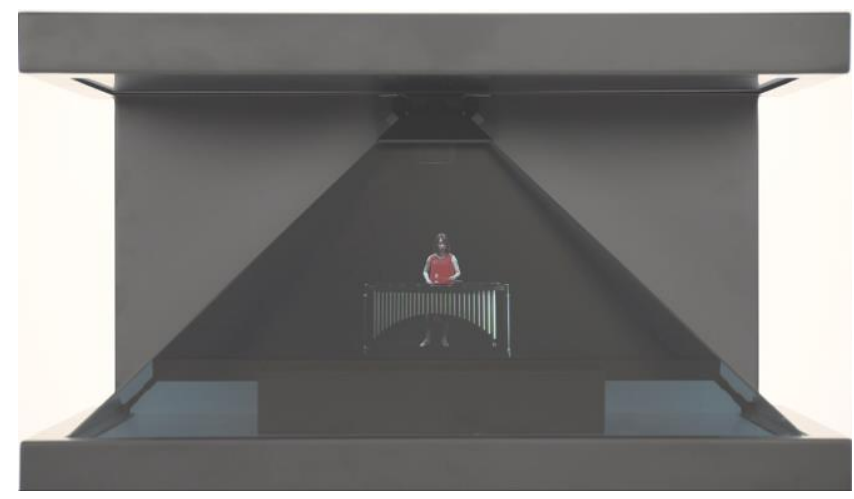

Figure 2: Holographic display

Improving the Sense of Presence We recorded all the performance sounds with the aim of increasing the sense of a presence. For the recording, we used a studio with very little reverberation; only the reverberation of the preceding sound enters the beginning of the melody segment because the melody is split into segments. Reverberation is added when the melody is played. We installed three pairs of speakers, and we set the pan pot and reverb for each direction so that the hologram seemed as realistic as possible (Figure 3). With the user's head placed between two pairs of speakers, both the sound and video enhance the sense of there being a presence.

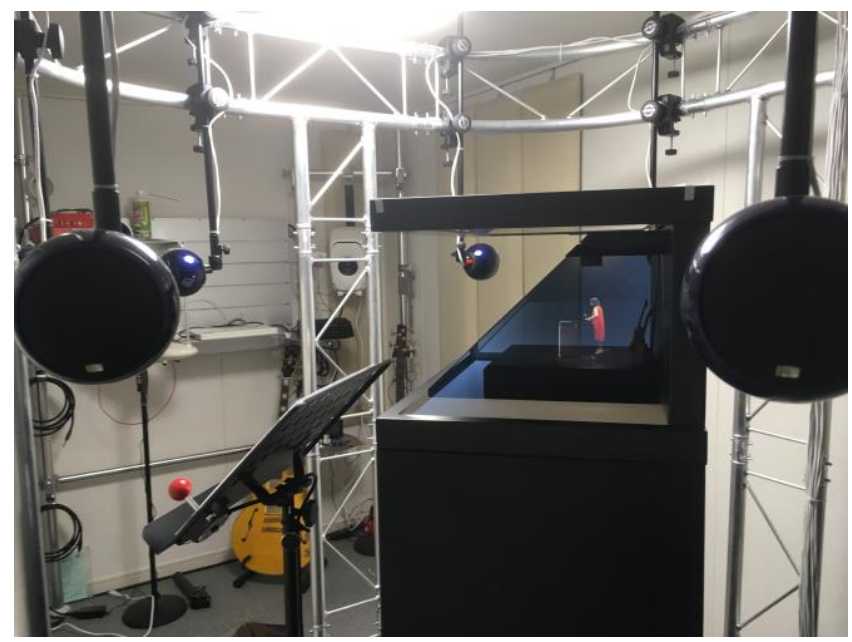

Figure 3: Three pairs of speakers

We built the Melody Slot Machine to enable users to freely control a holographic virtual performer. Band-out-of-the-Box enables customized interactions between a live, improvising musician and the computer [5]. The Virtual Musical Multi-Agent
System [6] and OMax Brothers software environment [7] enable improvisation with a human performer based on a multi-agent architecture. The continuator makes it possible to search for melodies that are an accurate continuation of a melody played by human performers using augmented Markov models [8]. The Guitarist Simulator enables a jam session with a virtual performer, which statistically learns a reaction model from a real human player [9]. The outputs of these systems are only performance sounds, and the performers are not visible.

VirJa Session enables performers to be visualized [10], although the visualizations are synthesized with computer graphics that can only show simple motions, such as a foot tapping or body rocking. Melody Slot Machine uses footage of performers taken with a video camera.

Melody-summarization [11], performance-rendering [12], and melody-prediction [13] systems all use time-span trees of the GTTM. The melody-prediction system provides real-time interaction and assists novices with musical improvisation by displaying the predicted notes on a piano lid. When novices find it difficult to continue playing the melody, they can continue the improvisation by playing a note displayed on the lid without impairing tonality.

Not everyone can use a melody-prediction system because it requires a piano keyboard. However, anyone can use Melody Slot Machine because it uses a melody-morphing method that involves rotating a dial.

We also developed a melody-morphing method based on the GTTM, which allows even beginner musicians to manipulate the melody using a time-span tree [14, 15]. Because of the low accuracy of the time-span tree analyzer of the GTTM [2-4, 13-15], our melody-morphing method has not been automated, and no composition has been performed using this method. Therefore, three composers capable of GTTM analysis attempted to use our melody-morphing method to compose melodies for this study.

The purpose of the musical dice game is to combine the melody selected by the dice in order [16]. Melody Slot Machine was inspired by the dice game idea in terms of changing the melody. However, the melody-composition methods for Melody Slot Machine and the musical dice game are very different. Melodies in the dice game are designed very elaborately so that they sound natural in any order. Our melody-morphing method uses the result of music structural analysis from the GTTM. Even if the melody is changed, the essential structure is the same and only ornamentation notes change.

The paper is organized as follows. Section 2 explains melodymorphing method, and Section 3 explains the implementation of Melody Slot Machine. Section 4 explains how we evaluated it, and Section 5 concludes with a summary and a brief overview of future work.

\section{MELODY MORPHING METHOD}

Melodies $A, B$, and $C$ and the melody-morphing method must meet the following conditions. Conditions 1 and 2 are for melody $C$ and conditions 3 and 4 are for the melody-morphing method. 
1. The similarity between $A$ and $C$ is closer than that of $A$ and $B$ and the similarity between $B$ and $C$ is closer than that of $A$ and $B$.

2. When $B$ is the same as $A, C$ will be the same as $A$.

3. The output of multiple $C$ 's depends on the parameters that determine the level of influence of the features of melodies $A$ and $B$.

4. $C$ will be a monophony if $A$ and $B$ are monophonies.

Our melody-morphing method uses time-span trees obtained from the results of GTTM analysis. The GTTM consists of four modules, each of which assigns a separate structural description to a listener's understanding of a piece of music. As Figure 4 shows, the four modules output a grouping structure, metrical structure, time-span tree, and prolongational tree. The time-span tree is a binary tree, a hierarchical structure that represents the relative structural importance of notes, which differentiate the essential parts of a melody from ornamentation.

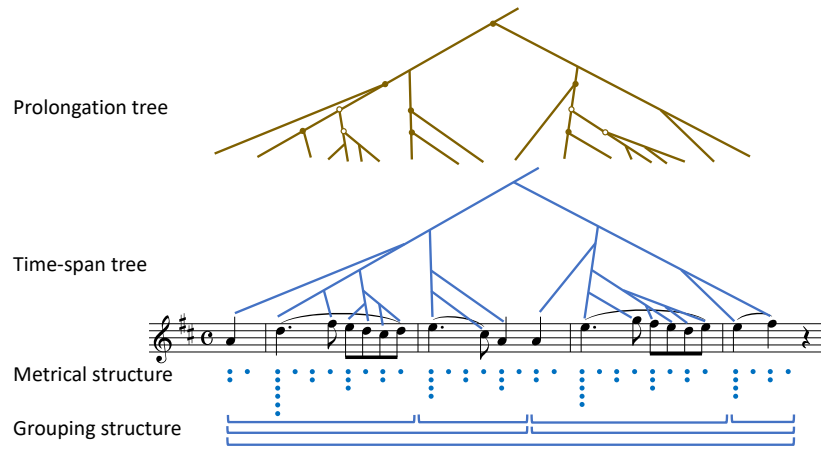

Figure 4: Structures obtained from GTTM analysis

\subsection{Abstraction of Melody}

Figure 5 shows an example of how a melody is abstracted using a time-span tree. The figure includes a time-span tree from melody D that demonstrates the results of the GTTM analyses. Important notes are connected to branches nearer the root of the tree, whereas unimportant notes are connected to leaves. We obtain an abstracted melody, E, by slicing the tree in the middle (line E) then omitting notes that have branch connections below line E. In the same manner, if we slice the tree higher up at line F, we can obtain an even more abstracted melody, F. We can regard this abstraction as a type of melody morphing because melody $\mathrm{E}$ is between melodies D and F.

\subsection{Primitive Operation of Time-span Trees}

To implement melody morphing, we use these primitive operations: the subsumption relationship (written as 드), meet (written as $\Pi$ ), and join (written as $\sqcup$ ) [11]. As shown in Figure $6 \mathrm{a}$, ᄃ represents the relationship by which "an instantiated object subsumes an abstract object." For example, the relationship between TD, TE, and TF, which are the time-span trees (or reduced time-span trees) of melodies D, E, and F in Figure 5, respectively, can be represented as follows.

$\mathrm{TF} \sqsubseteq \mathrm{TE} \sqsubseteq \mathrm{TD}$
Figure $6 \mathrm{~b}$ illustrates the meet operator, which extracts the largest common part or the most common information of the time-span trees of two melodies in a top-down manner. Finally, Figure $6 \mathrm{c}$ illustrates the join operator, which joins two time-span trees in a top-down manner as long as their structures are consistent.

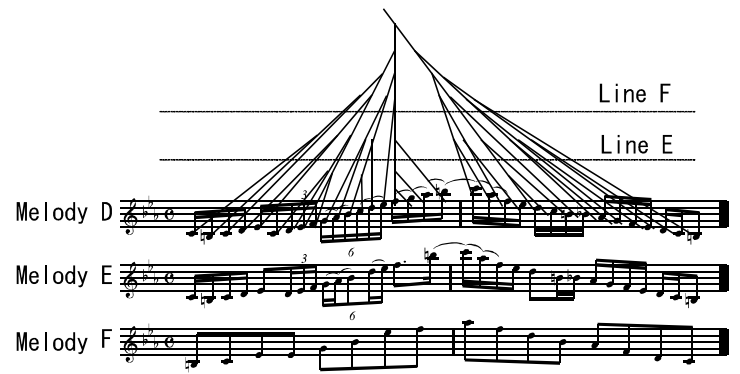

Figure 5: Abstraction of melody (a)

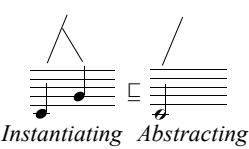

(b)

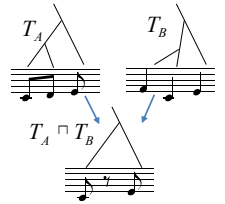

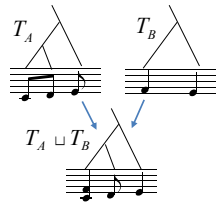

Figure 6: Subsumption relationship 드, meet $\Pi$, and join $\sqcup$ operations

\subsection{GTTM-based Melody Morphing}

In this subsection, we explain our GTTM-based melody morphing method [14, 15].

Morphing changes one image into another through a seamless transition. For example, a morphing method for a facial image can create intermediate images through the following operations.

1. Linking characteristic points such as the eyes and nose in two images, as shown in Figure 7a.

2. Rating the intensities of shape (position), color, and so forth in each image.

3. Combining the images.

(a)
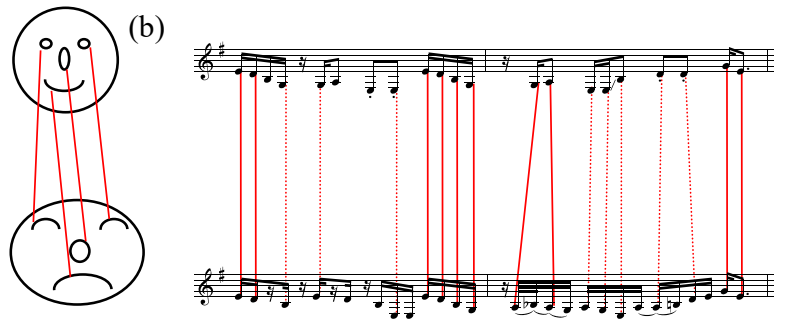

Figure 7: Examples of linking two images and two
melodies

Similarly, our melody-morphing method creates intermediate melodies through the following operations. 
1. Linking the most common information of the time-span trees for two melodies, as shown in Figure $7 \mathrm{~b}$.

2. Abstracting the notes of a melody in a differing branch of the time-span tree by using reduction steps for melody divisions with the method.

3. Combining both melodies.

\subsubsection{Linking Common Information}

By using the respective time-span trees $T_{A}$ and $T_{B}$ from melodies $A$ and $B$, we can calculate the most common information $T_{A} \sqcap T_{B}$, which includes the essential parts of both A and B (Figure 8). The meet operation $T_{A} \sqcap T_{B}$ abstracts common notes from $T_{A}$ and $T_{B}$, and the discarded notes are then regarded as the difference information of $T_{A}$ and $T_{B}$.

When calculating $T_{A} \sqcap T_{B}$ by extracting the largest common part of $T_{A}$ and $T_{B}$ in a top-down manner, the result may change depending on whether octave notes such as $C 4$ and $C 3$ can be distinguished. If we distinguish octave notes, then $C 4 \sqcap C 3$ will be empty, denoted as $\perp$. However, if we do not distinguish octave notes, the result is just $C$, which abstracts the octave information. We regard a note and its octave as different notes because processing is difficult if the octave information is undefined.

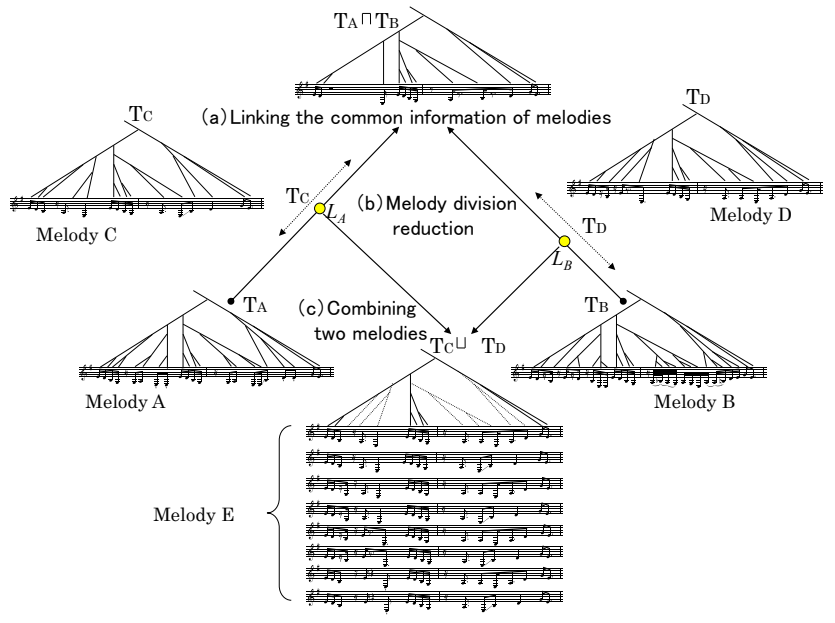

Figure 8: Our melody-morphing method

\subsubsection{Melody Divisional Reduction.}

Next, we argue that the difference information of $T_{A}$ and $T_{B}$ includes features not present in the other respective melodies. Therefore, we need a step for smoothly inserting or re-moving such features. The reduction step of our melody-morphing method abstracts the notes of the melody in a difference branch of the time-span tree by applying the abstraction described in Sub-subsection 2.3.1.

With this step, we can acquire melodies $C m(m=1,2, \ldots, n)$ from $T_{A}$ and $T_{A} \sqcap T_{B}$ with the following algorithm. Subscript $\mathrm{m}$ indicates the number of notes in the difference information of the time-span trees that are included in $T_{C m}$, but not in $T_{A} \sqcap T_{B}$.

Step 1: Determine level of abstraction. The user selects a parameter $L$, which determines the level of abstraction for the melody. This parameter can range from 1 to the number of notes in the difference information of the time-span trees included in $T_{A}$ but not in $T_{A} \sqcap T_{B}$.

Step 2: Abstract notes in difference information. The note with the fewest dots in the difference information is selected and abstracted. The number of dots can be acquired from the GTTM analysis results. If two or more notes are tied for the lowest number of dots, we select the first one by reading the music from left to right.

Step 3: Iterate; Step 2 is iterated L times. The subsumption relationships hold as follows for the time-span trees constructed with the aforementioned algorithm.

$$
T_{A} \sqcap T_{B} \subsetneq T_{C n} \subsetneq T_{C n-1} \subsetneq \ldots \subsetneq T_{C 2} \subsetneq T_{C 1} \subsetneq T_{A} \text {. }
$$

In Figure 9, nine notes are included in $T_{A}$ but not in $T_{A} \sqcap T_{B}$. Therefor, the value of $n$ is 8 , and we can obtain eight intermediate melodies $\mathrm{Cm}(m=1,2, \ldots, n)$ between $T_{A}$ and $T_{A} \Pi$ $T_{B}$. Because of this, melody $\mathrm{Cm}$ attenuates features that occur in melody $A$, but not in $B$. In the same manner, we can obtain melody $D$ from $T_{B}$ and $T_{A} \sqcap T_{B}$ as follows:

$T_{A} \sqcap T_{B}$ ᄃ $T_{D}$ ᄃ $T_{B}$.

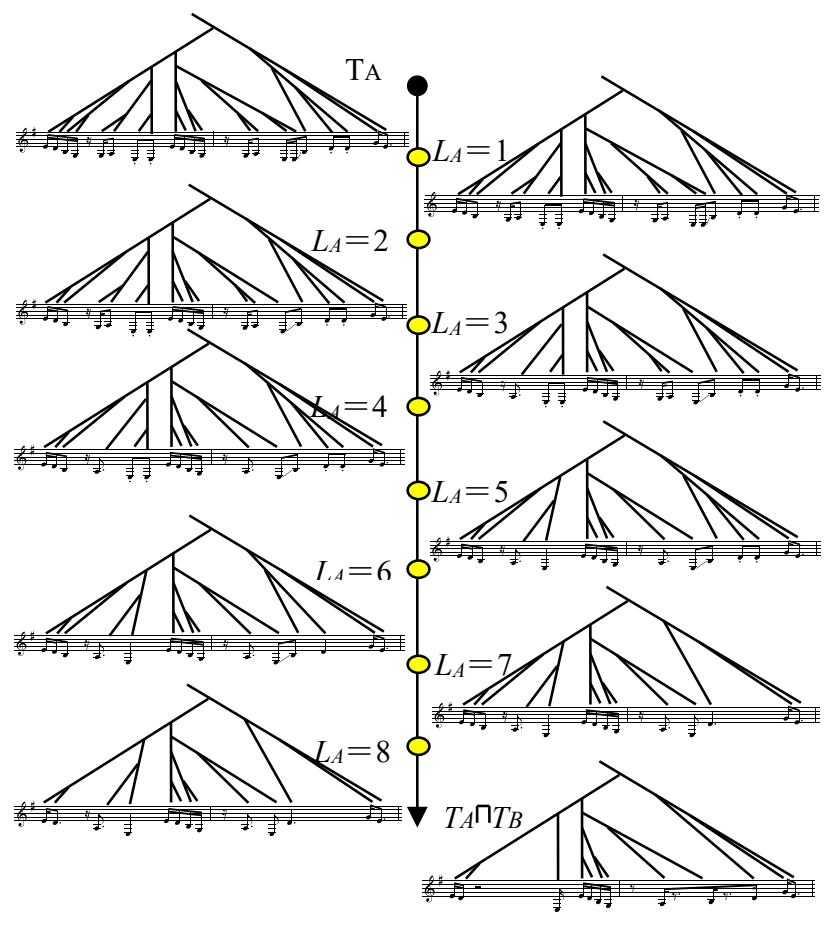

Figure 9: Melody divisional reduction

\subsubsection{Combination of Two Melodies}

Finally, we used the join operator to combine melodies $C$ and $D$, which are the results of divisional reduction using the timespan trees of melodies $A$ and $B$. The simple join operator is not 
sufficient for combining $T_{C}$ and $T_{D}$ because $T_{C} \sqcup T_{D}$ does not always exhibit monophony, even if $T_{C}$ and $T_{D}$ are monophonic.

To solve this problem, we introduced a special operator [ $n 1$, $n 2$ ], which indicates either note $n 1$ or note $n 2$ because $n 1 \sqcup n 2$. The results of $T_{C} \sqcup T_{D}$ are all monophonic combinations given by the operators. In the following description, we omit $T$.

\subsection{Adjustment by Musicologist}

Although we plan to automate our melody-morphing method, we cannot use it without a musicologist at this time. In the experiment described in Section 4, musicologists did the following three actions. First, they analyzed the melody to acquire an appropriate time-span tree. Second, they decided the order in which the notes would be abstracted in the melody divisional reduction in Sub-section 2.3.2. Third, they made adjustments manually.

After the first and second manual processes by musicologists, the morphed melody can be acquired automatically. However, using this method to morph melodies sometimes results in too few changes. Therefore, the musicologists made adjustments manually when the morphed melodies acquired with the method were not changed enough. The details of the adjustment are described in Section 4.

\section{IMPLEMENTATION}

The holographic display (Dremoc HD3) can be viewed from three directions because of three glass panes with semitransparent film and a reflecting display installed on top of the device. The holographic display is most realistic when viewed from a horizontal angle; it seems as if real miniature performers are present. Figure 10 shows the hardware implementation of Melody Slot Machine.

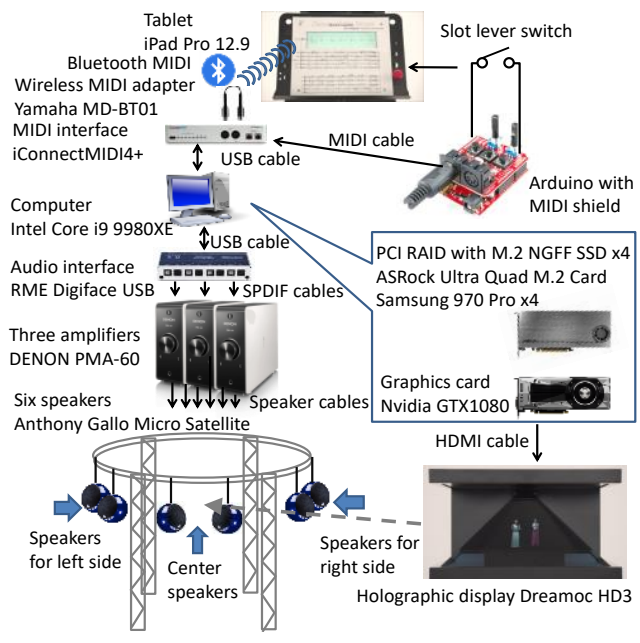

Figure 10: Implementation

\subsection{User Interface}

The user operates the slot dial on the tablet (iPad Pro 12.9) and the slot lever attached to the music stand. The tablet and computer communicate using the Bluetooth MIDI through a MIDI interface and wireless MIDI adapter. Bluetooth MIDI is widely broadcast to wireless devices as a standard MIDI signal on Bluetooth, and a connection can be made via MIDI cable if connecting via Bluetooth proves difficult.

The position of the slot dial, which dictates which melody to play, is transmitted from the tablet to the computer. Conversely, the computer notifies the current playback position of the piece to the tablet, and the playback position is displayed on the tablet. The slot lever is a switch, and the connection status of the switch is detected using a microcomputer (Arduino). It is connected to the computer through the MIDI shield and MIDI interface (iConnectMIDI4+).

\subsection{Audio Processing}

We installed speakers to cover the proper head position in all three directions to encourage users to view the holographic display at the best position possible. We used Anthony Gallo Micro Satellite speakers, which have high quality sound even at low volumes. The sound is converted to three stereo SPDIF signals by using an audio interface (RME Digiface USB), which is connected to the computer then connected to three amplifiers.

The system is controlled using the Max/MSP on the computer. The sound files are recorded in advance for each track and divided into segments, and the saved files are used. When the user sets the dial numbers on the tablet, the files are played in the order concatenated on the max/MSP. Mastering parameters are set for each of the three directions with a plug-in installed on the computer, so that it feels as if the sound is produced by the performer on the holographic display.

\subsection{Video Processing}

The video signal is connected to the holographic display with an HDMI cable via the graphics card. The video data are collected in advance from three directions for all melody tracks. When the user sets the dial numbers on the tablet, the system plays according to those numbers. The video file size is larger than the audio, and it takes more time to start playing the file; making it more complicated than sound processing.

First, all the video files are concatenated into one file, then two copies are written to disk as file 1 and file 2. Next, during the playback of video $A$ in file 1 , if the next video to be played back is B, file 2 seeks the playback position of video B, and playback occurs immediately. At the moment video A ends, it releases the connection of the renderer connected to file 1 , connects to file 2 , and plays video $\mathrm{B}$. The switching of this renderer ends within one frame, which is within 33.3 milliseconds, so a smooth connection without dropped frames is apparent.

We implemented this algorithm by using a VIDDULL video engine in the Max/MSP and Apple ProRes 422 video codec with a frame rate of 30 [fps]. 


\section{EXPERIMENT AND RESULTS}

We asked three musicologists to generate intermediate melodies. The musicologists had over 10 years of experience in GTTM analysis and a deep understanding of our melodymorphing method. We did not decide the length or name of the input pieces, and the musicologists created intermediate melodies based on the melody-morphing method with their own ideas.

\subsection{Mozart's Twelve Variations from "Ah! vous dirai-je, maman,” K. 265/300e}

The first musicologist selected the theme and Variation No. 1 of Mozart's Twelve Variations from "Ah! vous dirai-je, maman," K. 265/300e, and constructed nine intermediate melodies between the two melodies. Figures 11 shows the first set of eight bars for the theme, Variation No. 1, and 9 intermediate melodies, out of 40 bars in total. Non-parenthetical notes were generated with the melody-morphing method, whereas parenthetical notes were added by the musicologist. Table 1 lists the numbers of total notes, notes generated with the melody-morphing method, and notes added by the musicologist for each melody.

Notes were added in five ways:

a) adding appoggiaturas, auxiliary notes, and passing notes.

b) borrowing a note from a neighboring branch or swapping the order of notes in a branch.

c) dividing a note into two notes of the same pitch.

d) expanding or contracting the melody.

e) quoting a melody.

In Figure 11, (a) illustrates morphing melodies 3 and 5 as examples of adding passing notes, whereas (b) is an example of swapping the order of the notes from Variation No. 1. Figure 11 (c) is an example of dividing a note into two notes of the same pitch; (d) is an example of expanding a melody, specifically the last two notes in the fourth bar of Variation No. 1; and (e) is an example of quoting a melody from the fifth and sixth bars of Variation No. 1.

All the notes in morphing melodies 1 and 2 were generated with the melody-morphing method because both melodies were close to the common information of the theme and Variation No. 1 , so melodies could be generated by simply choosing an appropriate note from each special operator without adding any sound. All the notes in morphing melody 5 were generated with the melody-morphing method because this melody was very close to a melody obtained by divisional reduction of Variation No. 1 , so all the notes were eighth notes.

In morphing melodies 1,2 , and 3 (the three melodies closest to the theme), only $1.5 \%$ of the notes were added by the musicologist. In contrast, morphing melodies 8 and 9 (the closest to Variation No. 1) had $37.9 \%$ of notes added by the musicologist. Notes needed to be added to prevent unnatural melodies when generating multiple melodies between a melody with many eighth notes, such as morphing melody 5, and a melody with many sixteenth notes, such as Variation No. 1.
In total, $78.5 \%$ of the notes were generated with the melodymorphing method, whereas the remaining $21.5 \%$ were added by the musicologist.

Table 1: Numbers of notes in each melody

\begin{tabular}{|lccc|}
\hline & $\begin{array}{l}\text { Total } \\
\text { number } \\
\text { notes }\end{array}$ & $\begin{array}{l}\text { Number of } \\
\text { notes } \\
\text { generated } \\
\text { with melody } \\
\text { morphing } \\
\text { method }\end{array}$ & $\begin{array}{l}\text { Number of } \\
\text { notes added by } \\
\text { musicologist }\end{array}$ \\
\hline Theme & 82 & - & - \\
\hline Morphing melody 1 & 51 & $51(100 \%)$ & $0(0 \%)$ \\
\hline Morphing melody 2 & 51 & $51(100 \%)$ & $0(0 \%)$ \\
\hline Morphing melody 3 & 93 & $90(96.8 \%)$ & $3(3.2 \%)$ \\
\hline Morphing melody 4 & 121 & $96(79.3 \%)$ & $25(20.7 \%)$ \\
\hline Morphing melody 5 & 94 & $94(100 \%)$ & $0(0 \%)$ \\
\hline Morphing melody 6 & 157 & $129(82.2 \%)$ & $28(17.8 \%)$ \\
\hline Morphing melody 7 & 176 & $157(89.2)$ & $19(10.8 \%)$ \\
\hline Morphing melody 8 & 253 & $164(64.8 \%)$ & $89(35.1 \%)$ \\
\hline Morphing melody 9 & 267 & $159(59.6 \%)$ & $108(40.4 \%)$ \\
\hline Variation No. 1 & 271 & - & - \\
\hline
\end{tabular}

\subsection{Mozart's Horn Concerto No. 1 and Ponchielli's Dance of the Hours from "La Gioconda"}

The second musicologist generated nine levels of intermediate melodies between Mozart's Horn Concerto No. 1 and Ponchielli's Dance of the Hours from "La Gioconda" for the input melodies. All the notes were generated with our melodymorphing method.

Figures 12 shows the Horn Concerto, Dance of the Hours, and four intermediate melodies from nine intermediate melodies. The time-span trees of four intermediate melodies in Figure 12 were analyzed after the musicologist generated the melodies.

We attempted to determine whether the method could generate interporative melody $M$ from melodies $A$ and $B$, which holds the following expression:

$$
\begin{aligned}
& \{R(A, M)<R(A, B) \text { and } R(A, M)<R(B, M)\} \text {, or } \\
& \{R(B, M)<R(A, B) \text { and } R(B, M)<R(A, M)\} \text {, }
\end{aligned}
$$

where $R(X, Y)$ indicates the similarity between melodies $X$ and $Y$.

To measure the similarity between melodies $X$ and $Y$, we used the following $R_{N}(X, Y)$ defined by Hirata [11], which indicates how much information was lacking from the two melodies because of the meet operation.

$R_{N}(X, Y)=\frac{|\operatorname{meet}(X, Y)|_{N}}{\max \left(|X|_{N},|Y|_{N}\right)}$,

where $|X|_{N}$ indicates the number of notes in melody $X$.

We used all pairs of sample melodies $A$ and $B$ from nine intermediate melodies. As a result, our melody-morphing method met condition 1 in Section 2. 


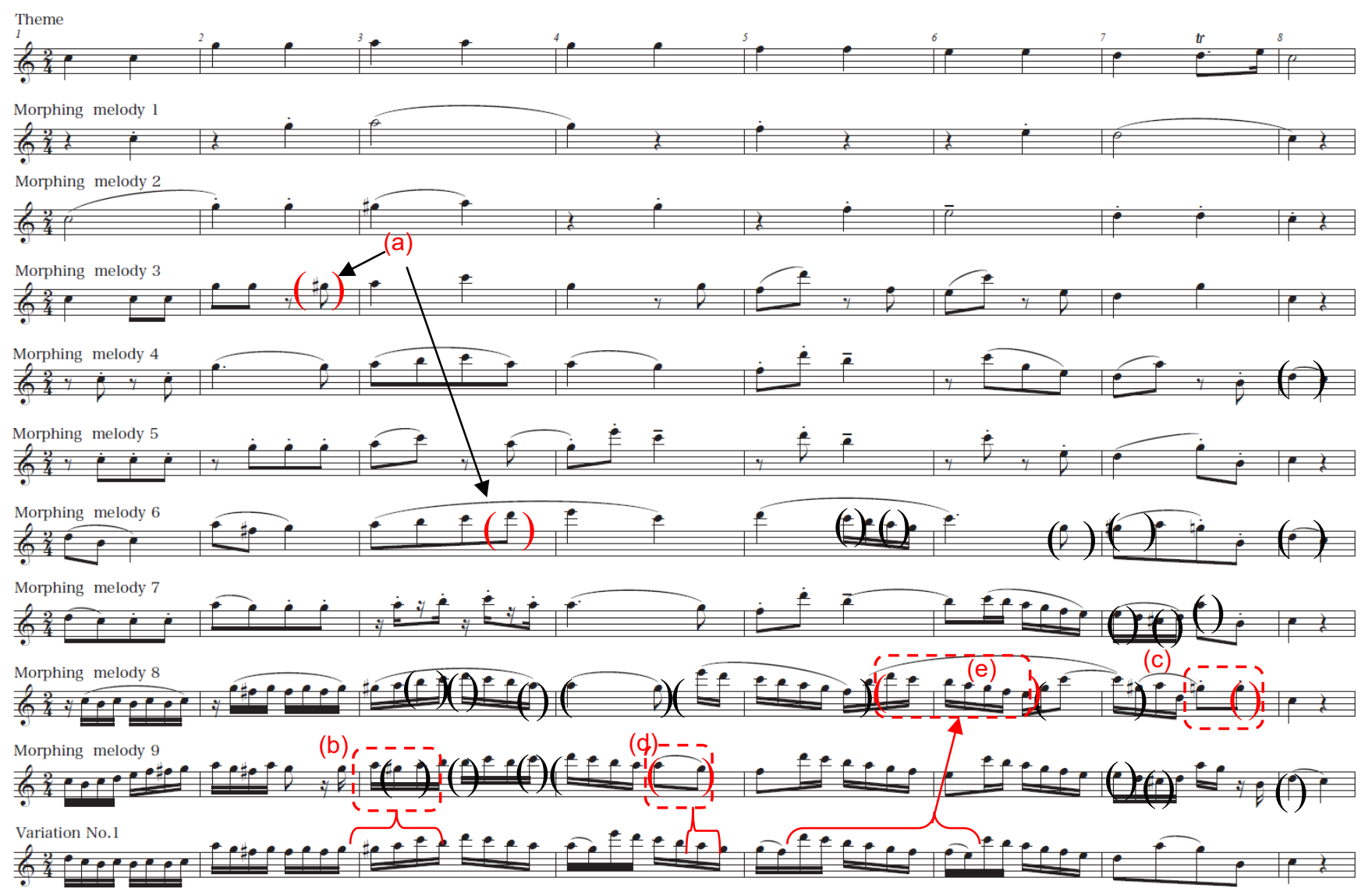

Figure 11: First eight bars of theme, Variation No. 1, and nine intermediate melodies

\subsection{Liszt's "La Campanella”}

The third musicologist generated five melodies $\left(M_{1}, M_{2}, M_{4}\right.$, $M_{5}$, and $M_{6}$ ) from Liszt's "La Campanella" by using melody morphing in a different manner than the other two, so that melody $M_{3}$ of "La Campanella" was in the intermediate, as shown by the following subsumption relationship.

$M_{1} \sqsubseteq M_{2} \sqsubseteq M_{3} \sqsubseteq M_{4} \sqsubseteq M_{5} \sqsubseteq M_{6}$

The subsumption relationships of Expression (6) were true in all melodies.

\subsection{Demonstration}

We demonstrated Melody Slot Machine in a creator's festival [17]. One hundred and fifty creators who passed the document review were given a booth of 1.6 square meters and exhibited their works. We displayed an image of a concert hall on the wall so that the user could feel like he/she was actually in a concert hall. We also used open-air headphones to prevent the sound from the speakers from affecting the adjacent booths (Figure 13). We used the morphing melody from Mozart's Horn Concerto No. 1 and Ponchielli's Dance of the Hours from "La Gioconda" because changes in the melody can be confirmed not only aurally but also visually from the movements of the marimba performer.

Three hundred people from a wide range of ages, from children to adults, half of whom were university students, took part in the demonstration. The children, who could not read scores, enjoyed the fact that the performance changed both visually and aurally by operating the dial. Those who could read the score tended to try various combinations of melodies for longer than 5 minutes and tended to look at the score on the dials without looking at the holographic display. A questionnaire survey was conducted to see if the performance was natural: $43 \%$ thought it was very natural, 37\% thought it was natural, $13 \%$ thought it was neither, $7 \%$ thought it was somewhat unnatural, and $1 \%$ thought it was very unnatural.

Since the accompaniment of the string quartet was repeated without change even if the dial was operated, some people may not have noticed the change in the marimba's melody immediately after the start of the demonstration. From advice to listen to the marimba, many noticed changes in the melody. Only two people did not notice that the melody of the marimba was changing due to dial operation because they could not hear the marimba performance separately from the accompaniment. 
Therefore, to fully experience this system, the user needs to be able to hear solo parts separately and reading the score is not always necessary.
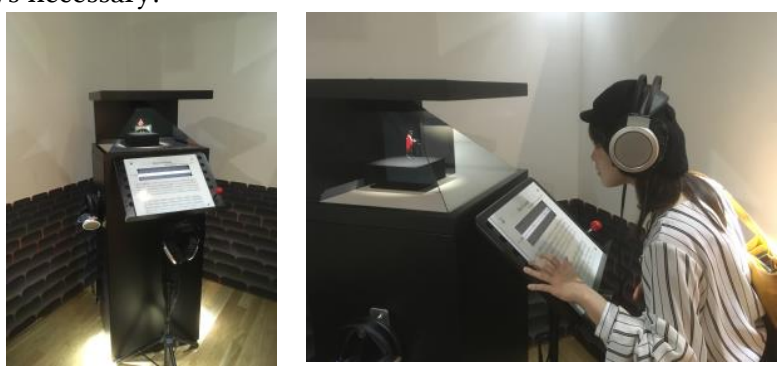

Figure 13: Melody Slot Machine at creators festival

\section{CONCLUSION}

We described the development of Melody Slot Machine. The following five points summarize the significance of this research. We:

1. developed a system in which the user can control a virtual player's performance. By adopting an interface that anyone can easily operate, such as a slot dial and lever, even beginner musicians can easily control the virtual player's performance.

2. displayed the holographic performer and built a highly realistic music system. Conventional virtual performers do not create a sense of reality because they are generated by only sound or are computer generated. The holographic display makes it possible to feel as if the performer is in front of you.

3. Three musicologists attempted to compose melodies using our melody-morphing method. The three musicians wrote a morphing melody despite the fact the melody-morphing method had never been used for composition. One musicologist generated $78.5 \%$ of the notes with the melody-morphing method, whereas the remaining $21.5 \%$ were manually added by her.

4. evaluated morphing melodies based on the similarity between two melodies $R_{N}(X, Y)$, which indicates how much information was lacking from the two melodies because of the meet operation. We confirmed that the similarity between $A$ and $C$ is closer than that of $A$ and $B$ and the similarity between $B$ and $C$ is closer than that of $A$ and $B$.

5. demonstrated Melody Slot Machine at a creators festival. We found that to fully experience this system, the user needs to be able to hear solo parts separately and reading the score is not always necessary.

For future work, we plan to create varus content for Melody Slot Machine. We also plan to generate virtual musicians, who can use the GTTM to compose and perform as humans. To achieve this, we will first develop an analyzer that automatically generates a high-precision time span tree then enable automatic melody morphing. Finally, enable melody operation and performances other than morphing using time span trees.

\section{ACKNOWLEDGMENTS}

This work was supported by JSPS KAKENHI, Grant Numbers $17 \mathrm{H} 01847$ and $16 \mathrm{H} 01744$.
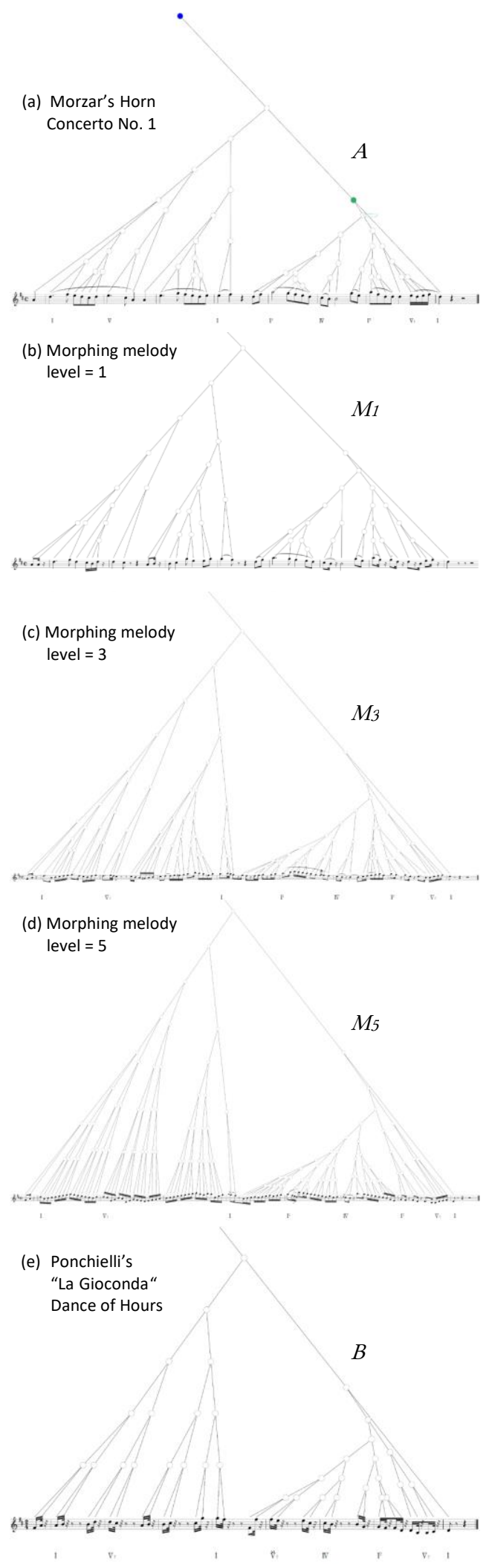

Figure 12: Morphing melodies of Horn Concerto and Dance of the Hours 


\section{REFERENCES}

[1] Fred Lerdahl and Ray S. Jackendoff, 1983. A Generative Theory of Tonal Music, MIT Press, Cambridge, Massachusetts, USA.

[2] Masatoshi Hamanaka, Keiji Hirata, and Satoshi Tojo. 2006 Implementing "A generative theory of tonal music," Journal of New Music Research 35 (4), 249-277. DOI: 10.1080/09298210701563238

[3] Masatoshi Hamanaka, Keiji Hirata, and Satoshi Tojo. 2016. бGTTM III: Learning-Based Time-Span Tree Generator Based on PCFG, Lecture Notes in Computer Science, Vol. 9617, pp. 387-404. DOI: 10.1007/978-3319-46282-0_25

[4] Masatoshi Hamanaka, Keiji Hirata, and Satoshi Tojo. 2018. deepGTTMIII: Multi-task Learning with Grouping and Metrical Structures, Lecture Notes in Computer Science, Vol. 11265, pp. 238-251. DOI: 10.1007/978-3030-01692-0_17

[5] Belinda Thom, 2003. Interactive Improvisational Music Companionship: A User-Modeling Approach, The User Modeling and User-Adapted Interaction Journal, Special Issue on User Modeling and Intelligent Agents, 13(1): 133-177. DOI: 10.1023/A:1024014923940

[6] Rodolfo Daniel Wulfhorst, Lauro Nakayama, and Rosa Maria Vicari, 2003. A Multiagent Approach for Musical Interactive Systems, In AAMAS '03: Proceedings of the second international joint conference on autonomous agents and multiagent systems, pp. 584-591. DOI $10.1145 / 860575.860669$

[7] Gérard Assayag, Georges Bloch, Marc Chemillier, Arshia Cont, and Shlomo Dubnov, 2006. OMax brothers: a Dynamic Topology of Agents for Improvization Learning, In AMCMM '06: Proceedings of the 1st ACM workshop on audio and music computing multimedia, pp. 125-132. DOI: $10.1145 / 1178723.1178742$

[8] Francois Pachet, 2003. The Continuator: Musical Interaction with Style, Journal of New Music Research, 32(3): 333-341. DOI: 10.1076/jnmr.32.3.333.16861
[9] Masatoshi Hamanaka, Masataka Goto, Hideki Asoh, and Nobuyuki Otsu, 2003. A Learning-Based Jam Session System that Imitates a Player's Personality Model, In Proceedings of the International Joint Conference on Artificial Intelligence (IJCAI2003), vol. 18, pp. 51-58.

[10] Masataka Goto, Isao Hidaka, Hideaki Matsumoto, Yosuke Kuroda, and Yoichi Muraoka, 1996. A Jazz Session System for Interplay among All Players - VirJa Session (Virtual Jazz Session System), In Proceedings of the 1996 International Computer Music Conference (ICMC1996), pp. 346-349.

[11] Keiji Hirata and Shu Matsuda, 2003. Interactive Music Summarization based on Generative Theory of Tonal Music, Journal of New Music Research, 32(2): 165-177. DOI: 10.1076/jnmr.32.2.165.16744

[12] Keiji Hirata and Rumi Hiraga, 2003. Ha-Hi-Hun plays Chopin's Etude. In Working Notes of IJCAI-03 Workshop on methods for automatic music performance and their applications in a public rendering contest, 2 pages.

[13] Masatoshi Hamanaka, Keiji Hirata, and Satoshi Tojo, 2008. Melody Expectation Method Based on GTTM and TPS, In Proceedings of the 9th International Conference on Music Information Retrieval conference (ISMIR2008), pp. 107-112.

[14] Masatoshi Hamanaka, Keiji Hirata, and Satoshi Tojo, 2008. Melody Morphing Method Based on GTTM, In Proceedings of the 2008 International Computer Music Conference (ICMC2008), pp. 155-158.

[15] Masatoshi Hamanaka, Keiji Hirata, and Satoshi Tojo, 2009. Melody extrapolation in GTTM approach, In Proceedings of the 2008 International Computer Music Conference (ICMC2009), pp. 89-92.

[16] Stephen A. Hedges, 1978. Dice Music in the Eighteenth Century, Music \& Letters, 59(2): 180-187.SICF20. 2019. Spiral Independent Creators Festival, http://www.sicf.jp/en/ 


\section{REFERENCES}

[1] Fred Lerdahl and Ray S Jackendoff, 1983. A Generative Theory of Tonal Music, MIT Press, Cambridge, Massachusetts, USA.

[2] MasatoshiHamanake, Keiji Hirata, and Satoshi Tojo. 2006. Implementing "A generative theory of tonal music," Journal of New Music Research 35 (4), 249-277. DOI: $10.1080 / 09298210701563238$

[3] Masatoshil..Hamanaka, Keiji...Hirata, and Satoshi...Tojo. 2016. бGTTM III Learning-Based Time-Span Tree Generator Based on PCFG, Lecture Notes in Computer Science, Vol. 9617, pp. 387-404. DOI: 10.1007/978-3-319-46282$0 \_25$

[4] MasatoshiHamanaka, Keiji Hirata, and SatoshilTojo. 2018. deepGTTM-III: Multi-task Learning with Grouping and Metrical Structures, Lecture Notes in Computer Science, Vol. 11265, pp. 238-251. DOI: 10.1007/978-3-030-01692$0 \_17$

[5] Belinda Thom, 2003. Interactive Improvisational Music Companionship: A User-Modeling Approach, The User Modeling and User-Adapted Interaction Journal, Special Issue on User Modeling and Intelligent Agents, 13(1): 133177. DOI: $10.1023 / \mathrm{A}: 1024014923940$

[6] Rodolfo DanielWulfhorst, Lauro Nakayama, and Rosa Maria Vicari, 2003. A Multiagent Approach for Musical Interactive Systems, In AAMAS '03 Proceedings of the second international joint conference on autonomous agents and multiagent systems, pp. 584-591. DOI: 10.1145/860575.860669

[7] Gérard Assayag, Georges Bloch, Marc Chemillier, Arshia Cont, and Shlomo Dubnov 2006. OMax brothers: a Dynamic Topology of Agents for Improvization Learning, In AMCMM '06: Proceedings of the 1st ACM workshop on audio and music computing multimedia, pp. 125-132. DOI $10.1145 / 1178723.1178742$
[8] Francois Pachet, 2003. The Continuator: Musical Interaction with Style Journal of New Music Research, 32(3): 333-341. DOI: 10.1076/jnmr.32.3.333.16861

[9] Masatoshi Hamanaka, Masataka Goto, Hideki Asoh, and Nobuyuki Otsu 2003. A Learning-Based Jam Session System that Imitates a Player's Personality Model, In Proceedings of the International Joint Conference on Artificial Intelligence (IJCAI2003), vol. 18, pp. 51-58.

[10] Masataka Goto, Isao Hidaka, Hideaki Matsumoto, Yosuke Kuroda, and Yoichi Muraoka, 1996. A Jazz Session System for Interplay among All Players VirJa Session (Virtual Jazz Session System), In Proceedings of the 1996 International Computer Music Conference (ICMC1996), pp. 346-349.

[11] Keiji Hirata and Shu Matsuda, 2003. Interactive Music Summarization based on Generative Theory of Tonal Music, Journal of New Music Research, 32(2): 165-177. DOI: $10.1076 / \mathrm{jnmr} .32 .2 .165 .16744$

[12] Keiji Hirata and Rumi Hiraga, 2003. Ha-Hi-Hun plays Chopin's Etude. In Working Notes of IJCAI-03 Workshop on methods for automatic music performance and their applications in a public rendering contest, 2 pages.

[13] Masatoshi Hamanaka, Keiji...Hirata, and Satoshi Tojo, 2008. Melody Expectation Method Based on GTTM and TPS, In Proceedings of the 9th International Conference on Music Information Retrieval conference (ISMIR2008), pp. 107-112.

[14] Masatoshi Hamanaka, Keiji Hirata, and Satoshi Tojo, 2008. Melody Morphing Method Based on GTTM, In Proceedings of the 2008 International Computer Music Conference (ICMC2008), pp. 155-158.

[15] Masatoshi. Hamanaka, Keiji...'Hirata, and Satoshi Tojo, 2009. Melody extrapolation in GTTM approach, In Proceedings of the 2008 International Computer Music Conference (ICMC2009), pp. 89-92.

[16] Stephen Hedges, 1978. Dice Music in the Eighteenth Century, Music \& Letters, 59(2): 180-187.SICF20. 2019. Spiral Independent Creators Festival, http://www.sicf.jp/en/ 